\title{
Hydrogel-Based Plasmonic Sensor Substrate for the Detection of Ethanol
}

\author{
Christoph Kroh ${ }^{1,2, *} \mathbb{C}$, Roland Wuchrer ${ }^{2}$, Nadja Steinke ${ }^{2}$, Margarita Guenther ${ }^{1}{ }^{(\mathbb{B}}$, \\ Gerald Gerlach ${ }^{1}$ (D) and Thomas Härtling ${ }^{1,2}$ \\ 1 Institute for Solid State Electronics, Technische Universität Dresden, Mommsenstraße 15, D-01062 Dresden, \\ Germany; mguenthe@mail.zih.tu-dresden.de (M.G.); gerald.gerlach@tu-dresden.de (G.G.); \\ thomas.haertling@ikts.fraunhofer.de (T.H.) \\ 2 Fraunhofer IKTS, Fraunhofer-Institute for Ceramic Technologies and Systems, Maria-Reiche-Straße 2, \\ D-01109 Dresden, Germany; roland.wuchrer@ikts.fraunhofer.de (R.W.); \\ nadja.steinke@ikts.fraunhofer.de (N.S.) \\ * Correspondence: christoph.kroh@tu-dresden.de
}

Received: 30 January 2019; Accepted: 7 March 2019; Published: 13 March 2019

\begin{abstract}
The in-line monitoring of ethanol concentration in liquids is a crucial part of process monitoring in breweries and distilleries. Current methods are based on infrared spectroscopy, which is time-consuming and costly, making these methods unaffordable for small and middle-sized companies. To overcome these problems, we presented a small, compact, and cost-effective sensing method for the ethanol content, based on a nanostructured, plasmonically active sensor substrate. The sensor substrate is coated with an ethanol-sensitive hydrogel, based on polyacrylamide and bisacrylamide, which induces a change in the refractive index of the substrate surface. The swelling and shrinking of such hydrogels offer a means to measure the ethanol content in liquids, which can be determined in a simple transmittance setup. In our study, we demonstrated the capability of the sensor principle for the detection of ethanol content ranging from 0 to $30 \mathrm{vol} \%$ ethanol. Furthermore, we determined the response time of the sensor substrate to be $5.2 \mathrm{~min}$, which shows an improvement by a factor of four compared to other hydrogel-based sensing methods. Finally, initial results for the sensor's lifetime are presented.
\end{abstract}

Keywords: plasmonics; ethanol sensor; hydrogel; nanostructure; hydrogel swelling; refractive index sensor; in-line sensor

\section{Introduction}

Efficient and low-cost sensors for the detection of chemical parameters in liquids are facing an ever-increasing demand in many different application fields, such as medical care or the food industry. Ethanol content in particular is one major parameter for process monitoring in breweries or distilleries.

Current detection methods for ethanol are based on the indirect calculation of ethanol content by oscillating density meters [1], or on a spectral determination by means of infrared spectroscopy [2,3]. Such systems require a laboratory environment and are very time-consuming and costly, which makes them unaffordable for many small and middle-sized breweries. In comparison, mobile laboratory devices like hydrometers have poor resolution and a strong temperature dependency [4], which makes them unsuitable for an accurate monitoring of the ethanol content over a time period of 7-10 days-the typical time span of the fermentation process. Furthermore, it is preferable to detect the ethanol content directly inside the brewing tanks. This demands a small, compact, and robust sensor device, for a continuous and simultaneous in-line monitoring of parameter changes in a complex medium.

One approach to measuring the ethanol content is based on the use of ethanol sensitive hydrogels. Hydrogels are hydrophilic, non-soluble, cross-linked polymers that show a reversible decrease in 
volume as a response to a chemical parameter change (in our case increasing ethanol content) in their environment. Thus, interrogating the swelling-shrinking state of the hydrogel allows the ethanol content to be deduced. The volume change can be detected, for instance, by means of a pressure sensor [5-8], which demands rather thick hydrogel sensor layers ranging from 50 to $330 \mu \mathrm{m}$ in the dried state [5-7] and up to $500 \mu \mathrm{m}$ in the swollen state [8]. In [9], a quartz crystal microbalance (QCM) was coated with $0.4 \mu \mathrm{m}$ and $0.8 \mu \mathrm{m}$ thick polymer layers, respectively. Due to the dependence between the layer thickness and response behavior [10], the equilibrium state in these systems is typically reached only after more than $20 \mathrm{~min}[5,6,8]$. One way to improve the response time of hydrogel-based sensors is to interrogate the ethanol-induced swelling state optically. To this end, we combined an ethanol-sensitive hydrogel with a nanostructured gold sensor substrate. This nanostructured metal surface supports localized plasmon oscillations [11,12], which can be excited in a simple transmittance setup. The plasmon oscillations are sensitive to refractive index changes in the vicinity of the metal substrate, which in turn appear as spectral changes of the transmittance spectrum $[13,14]$. During the swelling and shrinking of the hydrogel induced by changing ethanol concentrations, water diffuses in or out of the gel, which affects the polymer chain-water ratio. This leads to a change in the effective refractive index on the metal-polymer boundary, and subsequently to spectral changes in the transmittance spectrum of the sensor substrate. The sensitivity of the substrates is restricted to a layer in the range of a couple of $100 \mathrm{~nm}$ thickness perpendicular to the surface only, which allows the lowering of the hydrogel layer thickness to $<1 \mu \mathrm{m}$. This will result in a shorter diffusion time of the analyte through the hydrogel layer, a faster establishment of the volume change equilibrium, and, consequently, a faster response time compared to other hydrogel-based sensor systems.

Ethanol-dependent swelling has already been reported for different hydrogel systems and sensor concepts $[15,16]$. In this work, the combination of a polyacrylamide-bisacrylamide-based ethanol-sensitive hydrogel and a nanostructured gold sensor substrate is introduced for the first time. The overall aim of this work is to develop a robust hydrogel-based sensor for the in-line measurement of ethanol, with an operational lifetime of more than 10 days. Therefore, we present our nanostructured sensor substrate, as well as the optical detection of different hydrogel swelling states induced by various ethanol contents. Furthermore, we report initial results concerning the long-term stability of this sensor substrate, which provide the first data for the desired sensor application in the brewing industry.

\section{Materials and Methods}

\subsection{Materials}

The substrate foil cycloolefin polymer (COP) was purchased from Microfluidic ChipShop (Jena, Germany). For the nanoimprint lithography, the photoresist SU-8 was obtained from micro resist technology (Berlin, Germany), the polydimethylsiloxane (PDMS) RTV-615 was purchased from KVD (Bad Wimpfen, Germany), and the silicon master containing the nanostructure was fabricated by Eulitha AG (Kirchdorf, Switzerland). For the surface functionalization of the sensor substrates, $\mathrm{N}, \mathrm{N}^{\prime}$-bis(acryloyl)cystamine (BAC) was purchased from Alfa Aesar. The monomer acrylamide (Aam) and the photoinitiator lithium phenyl-2,4,6-trimethylbenzoylphosphinate were purchased from Sigma Aldrich. The cross-linker N, $\mathrm{N}^{\prime}$-methylen-bis-acrylamide (BIS) was obtained from Carl Roth $\mathrm{GmbH}+$ Co. KG (Karlsruhe, Germany).

\subsection{Fabrication of the Sensor Substrate}

The plasmonic sensor substrate consisted of a $3 \times 3 \mathrm{~mm}^{2}$ nanopillar array in a hexagonal lattice, fabricated by means of nanoimprint lithography, according to Yang et al. [11]. A silicon master fabricated by means of UV lithography was used to transfer a negative of the nanostructure into a PDMS mold. The PDMS was imprinted on a UV-curable SU-8 photoresist, which was uniformly spread on the substrate carrier, using the microcontact printer $\mu \mathrm{CP} 4.1$ (GeSiM mbH, Großerkmannsdorf, 
Germany). To enable a homogeneous distribution of the photoresist, the wafer was heated to a temperature of $95{ }^{\circ} \mathrm{C}$ before imprinting. A 4 inch COP-foil wafer with a thickness of $188 \mu \mathrm{m}$ was used as a substrate carrier. Subsequently, the nanopillars were cured with UV radiation, and the PDMS mold was removed. The nanopillars had a diameter of $230 \mathrm{~nm}$, a center-to-center distance of $450 \mathrm{~nm}$, and a height of $150 \mathrm{~nm}$ (Figure 1). Finally, the substrates were coated with a $2 \mathrm{~nm}$ thick chromium adhesive layer, and a $40 \mathrm{~nm}$ gold layer for surface plasmon activation. The wafer was cut into square pieces with a side length of $10 \mathrm{~mm}$.

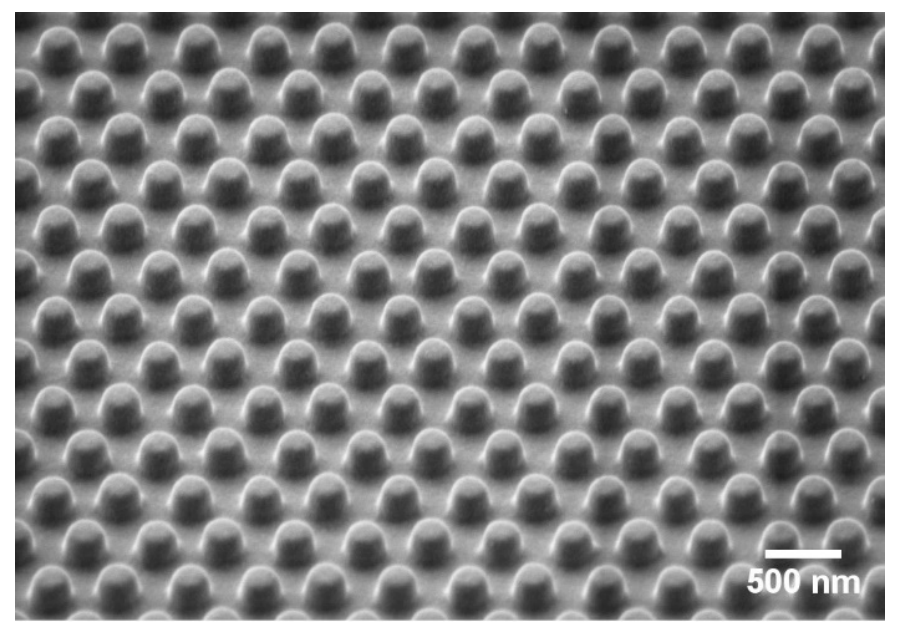

Figure 1. Scanning electron microscope image (SEM) of the nanostructured gold sensor substrate.

\subsection{Hydrogel Synthesis and Immobilization on the Sensor Substrate}

For the hydrogel immobilization, the sensor substrates were rinsed with ethanol and treated with UV/ozone (UV/ozone ProCleaner, NanoAndMore GmbH, Wetzlar, Germany) for 20 min. For a covalent binding of the hydrogel, the sensor substrates were treated with BAC (0.4 mmol) dissolved in dimethyl sulfoxide (DMSO).

The hydrogels were synthesized according to the protocol of Erfkamp et al. [8]. Firstly, 10 vol\% $(0.32 \mathrm{mmol})$ of acrylamide (Aam) and $0.44 \mathrm{~mol} \%(1.39 \mu \mathrm{mol})$ of bisacrylamide (BIS) were diluted in distilled water and degassed in a nitrogen atmosphere for $5 \mathrm{~min}$. Subsequently, 1 vol\% (32 $\mu \mathrm{mol})$ of the photo initiator lithium phenyl-2,4,6-trimethylbenzoylphosphinate (LAP) were added to the stock solution and mixed for $10 \mathrm{~min}$ in a nitrogen atmosphere. Each sensor substrate was coated in $10 \mu \mathrm{L}$ of the stock solution. Polymerization was finally carried out with UV light (wavelength range 320-500 $\mathrm{nm}$ ) for $2 \mathrm{~min}$. To remove residues of the monomer and the photo initiator, the sensor substrates were washed in distilled water for $48 \mathrm{~h}$. After the synthesis, the sensor substrates containing the hydrogel were placed alternately in pure ethanol and distilled water five times for conditioning.

\subsection{Studies of the Free Swelling of the Hydrogel}

For the investigation of the free swelling, $5 \mathrm{~mm}$ squares of the polyacrylamide (PAM) hydrogel were synthesized. After synthesis and conditioning, the initial length $l_{0}$ of the swollen hydrogel samples was determined by means of microscopy, with five-fold averaging. Subsequently, the shrinking of the gels was induced by incubation in a $50 \mathrm{vol} \%$ ethanol solution for two hours. After the determination of the shrunken length $l$ of the gels, the solution was changed back to distilled water. This process was repeated for several cycles. From the results, the swelling degree $S$ of the hydrogel was calculated as the relative change in length as follows [17]:

$$
S=\frac{l}{l_{0}} .
$$




\subsection{Plasmonic Transducer and Sensor Setup}

The sensors consisted of a nanostructured gold substrate, which is well known to support localized surface plasmon oscillations [11-13]. These collective electron oscillations can be excited in the nanostructured metal layer with normal incident light without additional optics. They cause a local electromagnetic field enhancement on the substrate surface within a range of a couple of $100 \mathrm{~nm}$ distance $[14,18]$. As described in many previous studies, these plasmon oscillations also lead to absorption and scattering of the incident light, which cause a local dip in the transmittance spectrum of the sensor substrate $[13,14]$. This dip, representing the plasmon oscillation resonance wavelength, subsequently results from a superposition of the incident LED spectrum and the extinction spectrum of the nanostructure, and shifts linearly with the refractive index in the vicinity of the sensor substrate $[19,20]$. This shift serves as the sensor signal and is independent of the intensity and initial position of the spectrum. Thus, such structures can be used as sensor elements for the detection of refractive index changes, and as a transducer element for hydrogels. The optical interrogation of the sensor substrate was accomplished with a simple home-built transmittance evaluation system. The substrates were bonded on an optical holder with PDMS and were irradiated with an LED (central wavelength $875 \mathrm{~nm}$, spot size $1 \mathrm{~mm}$ ) in the spectral window between 750 and $1000 \mathrm{~nm}$. The light transmitted through the sensor substrate was collected with an optical fiber and directed to a spectrometer (iHR550, Horiba Jobin Yvon GmbH, Unterhaching, Germany), which recorded the transmittance spectra with a resolution of $0.03 \mathrm{~nm}$ (diffraction grating with 1200 slits). After smoothing by means of an FFT filter, the resonance wavelength was calculated from a Gaussian fit of each transmittance spectrum. Figure 2 shows a schematic representation and a picture of the sensor setup.
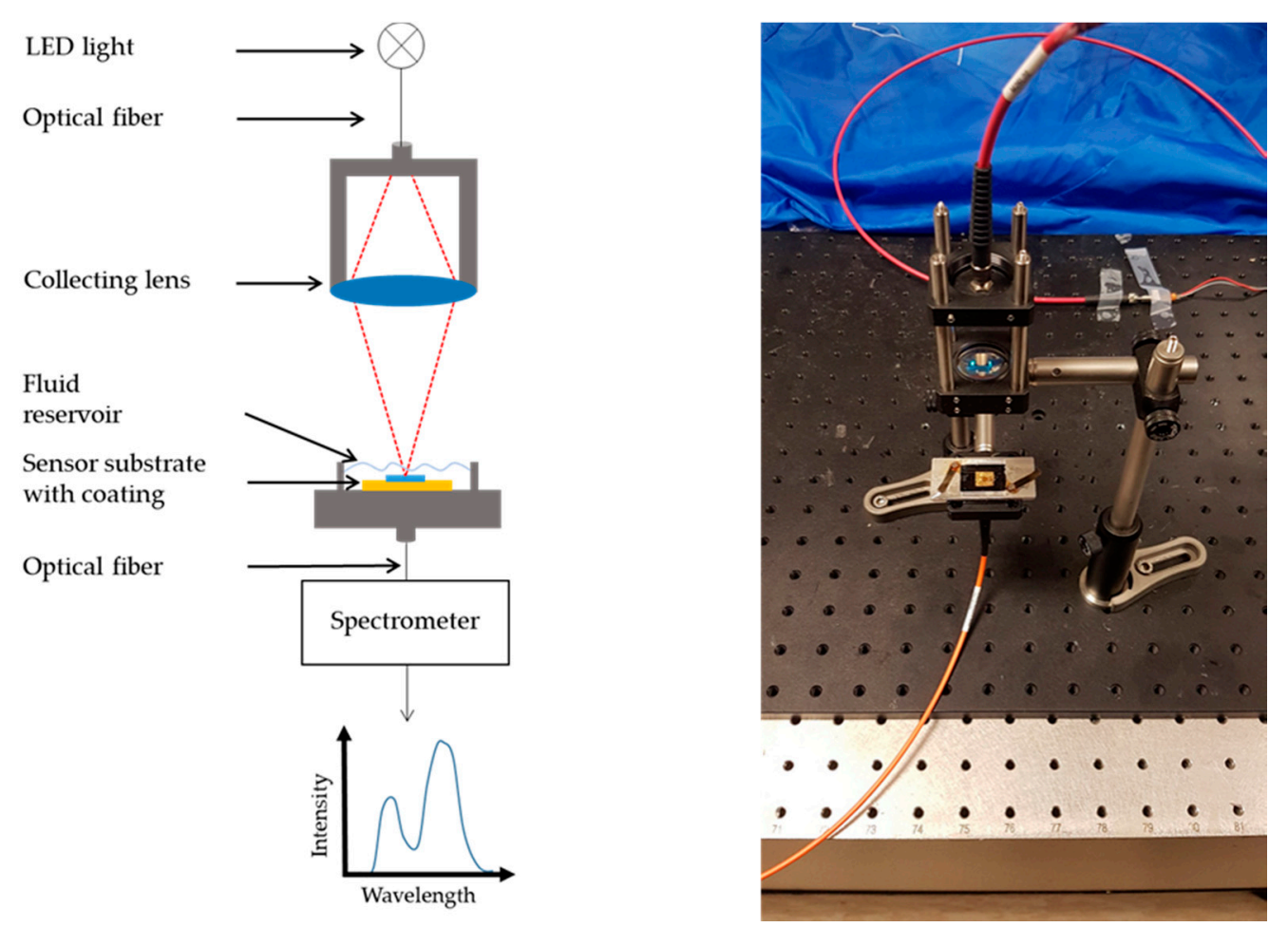

Figure 2. Schematic representation and picture of the sensor setup to record the transmittance spectra of the sensor substrates.

\section{Results and Discussion}

\subsection{Characterization of the Sensor Substrate}

As a first step, the quality of the sensor substrates, including the spectral position of the resonance wavelength and the refractive index sensitivity, was determined. In order to achieve this, the sensor 
substrates were placed in aqueous solutions with different ethylene glycol content (see Table 1) to analyze the refractive index sensitivity of the sensor substrate. The reference measurement was carried out by means of a refractometer (DR201-95, Krüss Optronic, Hamburg, Germany), with a measurement uncertainty of 0.0003 RIU (refractive index unit).

Table 1. Water-ethylene glycol solutions for the calibration measurement of the refractive index.

\begin{tabular}{cc}
\hline Solution & Refractive Index \\
\hline $100 \mathrm{v}^{\circ} \mathrm{H}_{2} \mathrm{O}$ & 1.3329 \\
$66 \mathrm{v} \% \mathrm{H}_{2} \mathrm{O}, 33 \mathrm{v} \% \mathrm{EG}$ & 1.3683 \\
$50 \mathrm{v} \% \mathrm{H}_{2} \mathrm{O}, 50 \mathrm{v} \% \mathrm{EG}$ & 1.3858 \\
$33 \mathrm{v} \% \mathrm{H}_{2} \mathrm{O}, 66 \mathrm{v} \% \mathrm{EG}$ & 1.4022 \\
$100 \mathrm{v} \% \mathrm{EG}$ & 1.4300 \\
\hline
\end{tabular}

A droplet $(20 \mu \mathrm{L})$ of each solution was placed on the sensor substrate and the respective transmittance spectrum was measured.

Subsequently, the refractive index sensitivity of the substrate was determined from the slope of a linear fit of the resonance wavelength. Figure 3 a shows the transmittance spectra for the different $\mathrm{H}_{2} \mathrm{O}-\mathrm{EG}$ solutions, while in Figure $3 \mathrm{~b}$ the correlation between the refractive index and plasmon resonance wavelength is depicted. The spectral position of the resonance wavelength in water was determined by means of a Gaussian fit (see Section 2.5) to $810 \mathrm{~nm}$. The spectra show a red-shift of the resonance wavelength with an increasing refractive index, and the sensitivity was calculated to $287 \mathrm{~nm}$ per RIU, which implies a resolution of the refractive index in the order of $10^{-4}$. The plotted uncertainty bars, ranging from $0.04 \mathrm{~nm}$ to $0.16 \mathrm{~nm}$, originated from the standard deviation of a five-fold averaging, in addition to the uncertainty of the Gaussian fit. The changes in the intensities of the spectra are caused by performance fluctuations and warming of the LED. Furthermore, absorption depends on the refractive index, which causes additional changes to the intensity. Hence, the resonance wavelength is independent of the intensity; such changes have no impact on the sensor signal and can consequently be disregarded.

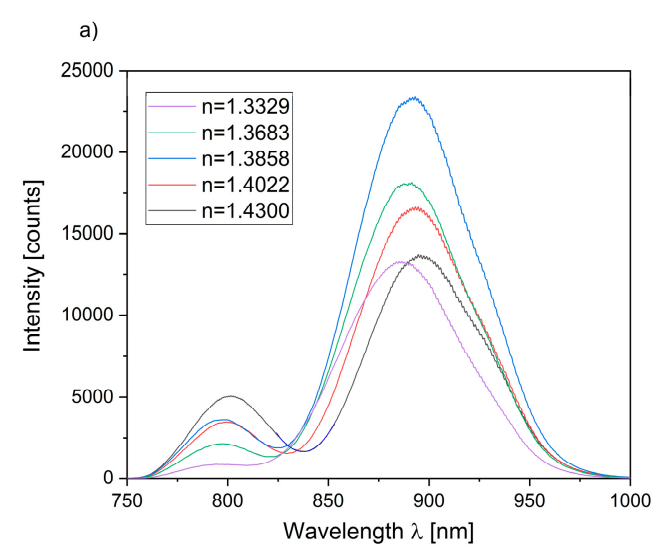

(a)

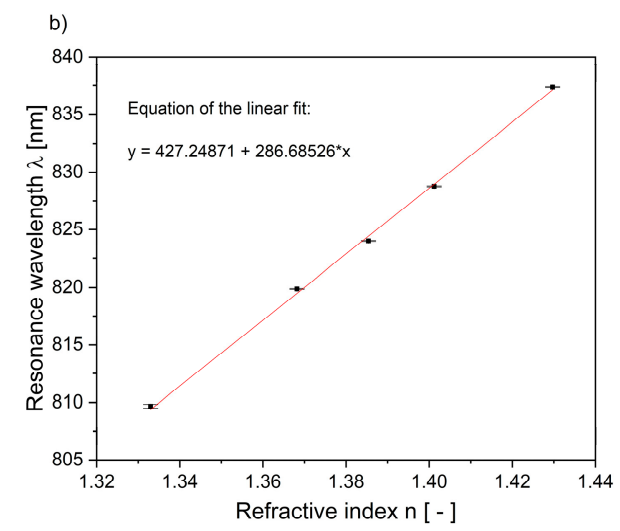

(b)

Figure 3. Interrogation of the sensitivity of the sensor substrates (a) Transmittance spectra of sensor substrates covered with water-ethylene glycol solutions of different refractive index n; (b) Linear shift of the resonance wavelength with increasing refractive index resulting in a sensitivity of $287 \mathrm{~nm} \mathrm{RIU}{ }^{-1}$.

\subsection{Optical Interrogation of Hydrogel Swelling States Induced by Ethanol}

\subsubsection{Reversible Free Swelling of the Hydrogel}

Hydrogels are well known for their reversible swelling process, which makes them interesting candidates for sensor materials. The swelling and shrinking mechanisms induced by ethanol were 
studied in [21,22]. After the addition of ethanol, the PAM hydrogel deswells. This can be explained by the influence of the alcohol on the water-polymer systems, whereby the free energy of the polymer-polymer contact increases, and the polymer chains attract each other. This causes a diffusion of water out of the gel and, subsequently, an increase of the refractive index inside the system. By decreasing the amount of ethanol, the free energy decreases and the gel swells back to its original state [21]. A further approach to describing the swelling mechanism of ethanol-sensitive hydrogels is based on the positive viscosity coefficient of ethanol, which causes stronger interactions inside the gel. These interactions again induce an attraction of the polymer chains, and a shrinking of the gel with increasing ethanol content [22].

Figure 4 shows the reversible free swelling of the PAM hydrogel over multiple cycles. The swelling degree of the hydrogel was determined according to the methods outlined in Section 2.4. The uncertainty bars originated from the standard deviation of the applied five-fold averaging.

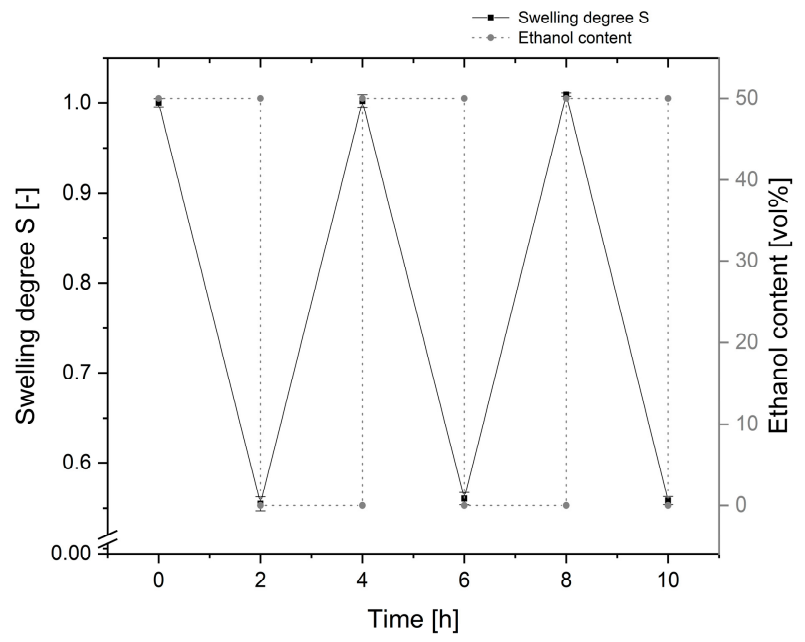

Figure 4. Reversible hydrogel swelling of the polyacrylamide (PAM) hydrogel versus ethanol content of the solution. The swelling degree $\mathrm{S}$ shows a decrease of about $45 \%$ between distilled water and a 50 vol\% ethanol solution.

After a change from 0 to $50 \mathrm{vol} \%$ ethanol content, the hydrogel showed a reversible shrinking of about $45 \%$ of its size over multiple cycles. This is in accordance with previous studies of the PAM-based hydrogel [8], and shows the suitability of such gels as a sensor material.

\subsubsection{Optical Detection of Different Hydrogel Swelling States}

The sensor substrates were coated with the PAM hydrogel and conditioned with water and ethanol solutions. To attain different swelling states, the sensor substrates were placed on the holder (see Figure 2), bonded with PDMS, and the fluid reservoir was filled with water containing different levels of ethanol content. After a $15 \mathrm{~min}$ immersion in each solution, the transmittance spectrum of the sensor substrate was taken by applying five-fold averaging. The resonance wavelength of the respective spectrum was determined with a Gaussian fit, and its average position was plotted over the respective ethanol content. For the optical monitoring of the swelling and shrinking, a hydrogel layer with a thickness of about $20 \mu \mathrm{m}$ was used. Figure 5 presents the position of the resonance wavelengths for an ethanol content between 0 and $30 \mathrm{vol} \%$. This position shows a red-shift with increasing ethanol content, which corresponds to an increasing effective refractive index of the gel. The plotted uncertainty bars originated from the standard deviation of the five-fold averaging, in addition to the uncertainty of the Gaussian fit. 


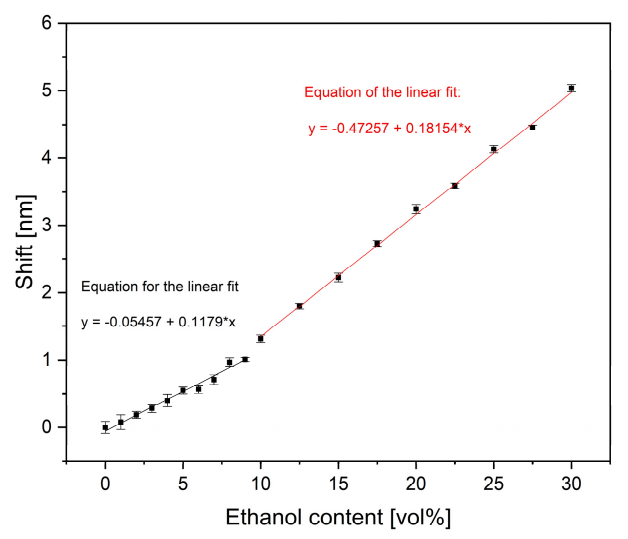

Figure 5. Shift of the resonance wavelength with increasing ethanol content due to the deswelling of the PAM hydrogel showing a linear behavior of the refractive index change.

The shrinking behavior of the hydrogel indicates a section-wise linear increase of the refractive index inside the hydrogel between 0 and $30 \mathrm{vol} \%$ ethanol content. We observed a clear shift of the resonance wavelength between 0 and $10 \mathrm{vol} \%$, with an interval of $1 \mathrm{vol} \%$. The slope of the shown data, representing the sensitivity of the sensor device, was determined as $0.118 \mathrm{~nm}$ per vol\% ethanol. With respect to the resolution of the spectrometer $(0.03 \mathrm{~nm})$, this would lead to a theoretical resolution of $0.3 \mathrm{vol} \%$ of ethanol and implies the suitability of the sensor substrate as a monitoring device in the brewing process. For higher ethanol concentrations, we observed a sensitivity of $0.182 \mathrm{~nm}$ per vol\% ethanol, which would lead to a theoretical resolution of $<0.2$.

We attributed the different sensitivities to a delayed starting behavior of the hydrogel swelling in low ethanol concentrations. This behavior, typical of the hydrogel, was described in previous works [8] and will require separate calibration curves depending on the desired application.

\subsubsection{Swelling Kinetics and Response Time}

In addition to the swelling behavior at different ethanol contents, a fast in-situ response is a crucial requirement of sensor devices. To determine the response time of the sensor material, the fluid reservoir was filled with water and the hydrogel was immersed for $30 \mathrm{~min}$. Afterwards, we investigated the hydrogel shrinking in a $20 \mathrm{vol} \%$ ethanol solution over $10 \mathrm{~min}$. Figure 6 shows the time-resolved shift of the resonance wavelength by means of the in-situ shrinking behavior of the PAM hydrogel, induced by a change in the ethanol content. The plotted uncertainty bars originated from the standard deviation of the Gaussian fit, which was used to determine the resonance wavelength.

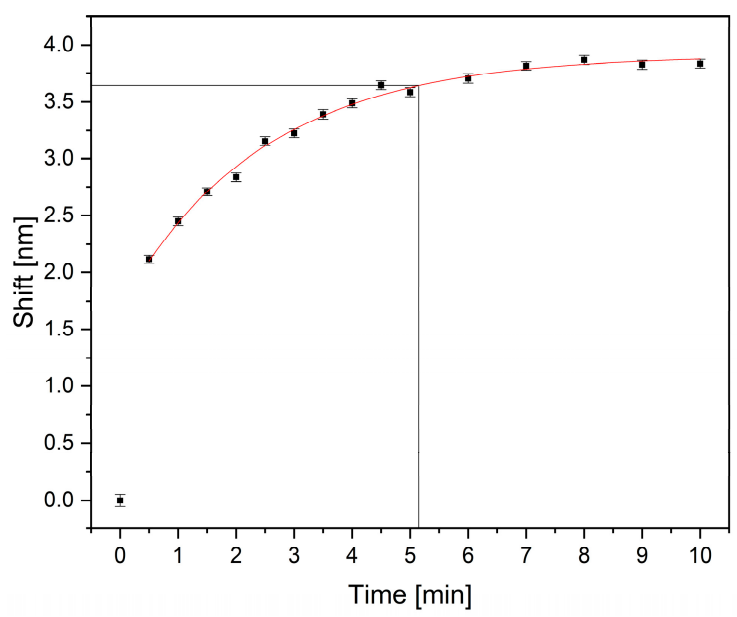

Figure 6. Time-resolved response of the sensor substrate covered with PAM hydrogel after a change from water to a $20 \mathrm{vol} \%$ ethanol solution. 
The results show an exponential increase of the resonance wavelength. It can clearly be seen that the gel reacts immediately upon ethanol contact, and more than $50 \%$ of the shift is reached after $0.5 \mathrm{~min}$. After $5.2 \mathrm{~min}, 95 \%$ of the steady-state value is reached, which shows an improvement by a factor of four compared to other hydrogel-based sensor systems $[5,6,8]$. This improvement is caused by the much thinner hydrogel layer, which in turn leads to a lower diffusion time of the fluid out of the gel and, subsequently, to a faster response to changing ethanol content.

\subsubsection{Long-Term Stability of the Sensor Substrate}

For use as an in-line sensing platform in the brewing process, a lifetime of 7-10 days is required for the sensor substrate. To initially investigate its long-term stability, the substrate was placed on the holder for 10 days and immersed in distilled water. Periodically, the liquid was changed to a $50 \mathrm{vol} \%$ ethanol solution, and the transmittance spectra were determined by a five-fold averaging both after each deswelling in ethanol and each distilled water immersion with constant swelling times. Afterwards, the ethanol solution was replaced again by distilled water. The resonance wavelengths were determined with a Gaussian fit.

Figure 7 presents the initial results for the long-term stability of the sensing material. The plotted error bars originated from the standard deviation of the five-fold averaging and of the uncertainty of the Gaussian fit.

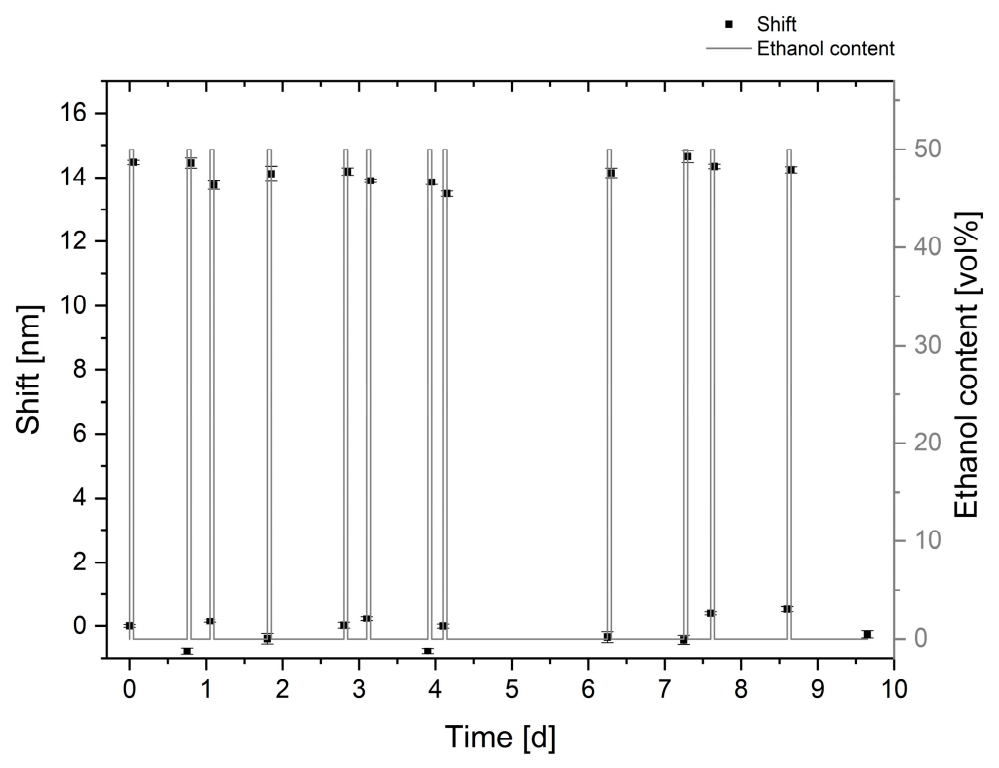

Figure 7. Signal stability of the resonance shift of the sensor substrate, coated with the PAM hydrogel, investigated over 10 days by a periodically induced deswelling in a $50 \mathrm{vol} \%$ ethanol solution.

The results show a shift in the resonance wavelength, induced by the shrinking of the hydrogel in ethanol of about $14 \mathrm{~nm}$, with no significant change over 10 days. Furthermore, the sensor substrate and the hydrogel were stable and no effects of ageing and delamination could be observed.

The deviations, we assume, could be caused by temperature variations during the experiment duration and the evaporation of the ethanol. However, this data proves the suitability of the surface functionalization for a bonding of the PAM hydrogel on the gold sensor substrates.

\section{Conclusions and Outlook}

In this paper, we reported on the suitability of an optical detection method for the determination of the ethanol concentration in aqueous solutions. We applied a nanostructured gold sensor substrate supporting localized surface plasmons as an optical transducer for an ethanol-sensitive hydrogel, to enable the detection of refractive index changes occurring during the swelling and deswelling of the 
gel. The combination of an optical transducer element and the PAM-based ethanol-sensitive hydrogel was studied for the first time.

The results showed a linear red-shift of the resonance wavelength in the nanostructure transmittance spectra, with increasing ethanol content due to the deswelling of the hydrogel, and a subsequently increasing refractive index on the transducer surface. It was shown that this shift resulted in a theoretical resolution of the ethanol content of about $0.4 \mathrm{vol} \%$. Furthermore, we showed an improvement in the response time in comparison to other hydrogel-based sensor systems by a factor of four, which led to a response time of about $5.2 \mathrm{~min}$. Additionally, we showed a sensor lifetime and a signal stability of more than 10 days, which would be sufficient for possible application in the brewing process.

However, it is still possible to improve the resolution of the sensor substrate, e.g., by modifying the hydrogel. As shown in [8], a decrease in the monomer or cross-linker content would lead to an enhanced deswelling of the gel, and subsequently to an increase of the sensitivity. However, this would cause a reduction in the mechanical stability of the gel, whereby an optimum between the latter and the sensitivity must be found. Another possibility to improve the sensitivity is the increase of the sensitivity of the optical sensor substrate itself.

Furthermore, the determined response time implies the suitability of the sensor substrate as an in-line process monitoring tool in the brewing process, where measurement intervals of $30 \mathrm{~min}$ are common. A further improvement in the response time can be obtained with even thinner hydrogel layers. For a plasmonic readout, a hydrogel layer thicknesses of around $1 \mu \mathrm{m}$ would be sufficient, which can be reached by the uniform or microstructured imprinting of the hydrogels. Future work will focus on the implementation of even thinner hydrogel layers by way of microstructuring, the optimization of the hydrogel sensitivity, and the cross-sensitivities of the gel to other parameters.

Nevertheless, the linear behavior, the high sensitivity, and the short response time of the plasmonic sensor system indicate its suitability as a platform for the in-line process monitoring of alcoholic fermentation in breweries.

Author Contributions: Conceptualization, C.K., R.W., N.S. and T.H.; Experiments, C.K.; Synthesis of the hydrogels, C.K.; Analyzes, C.K.; Writing original draft preparation, C.K.; Writing review and editing, G.G., T.H., M.G.; Supervision, M.G., G.G. and T.H.; Project administration, R.W.; Funding acquisition, G.G. and T.H.

Funding: This research was funded by DFG Research Training Group 1865 and the BMBF VIP+ program, grant number 03VP01970.

Acknowledgments: The authors thank GeSiM mbH, Großerkmannsdorf, Germany for their cooperation concerning plasmonic structure fabrication and Rüdiger Rosenkranz for the SEM work. Furthermore, the authors thank Jan Erfkamp for his cooperation concerning the hydrogel synthesis. Funding from the DFG Research Training Group 1865 and the BMBF VIP+ program under grant number 03VP01970 is gratefully acknowledged.

Conflicts of Interest: The authors declare no conflict of interest.

\section{References}

1. Gonzalez-Rodriguez, J.; Perez-Juan, P.; Luque de Castro, M.D. Determination of ethanol in beverages by flow injection, pervaporation and density measurements. Talanta 2003, 59, 691-696. [CrossRef]

2. Cavinto, A.G.; Mayes, D.M.; Ge, Z.; Callis, J.B. Noninvasive Method for Monitoring Ethanol in Fermentation Processes Using Fiber-Optic Near-Infrared Spectroscopy. Anal. Chem. 1990, 62, 1977-1982. [CrossRef]

3. Michel, K.; Bureau, B.; Boussard-Plédel, C.; Jouan, T.; Adam, J.L.; Staubmann, K.; Baumann, T. Monitoring of pollutant in waste water by infrared spectroscopy using chalcogenide glass optical fibers. Sens. Actuators 2004, 101, 252-259. [CrossRef]

4. Osorio, D.; Pérez-Correa, J.R.; Agosin, E.; Cabrera, M. Soft-sensor for on-line estimation of ethanol concentrations in wine stills. J. Food Eng. 2008, 87, 571-577. [CrossRef]

5. Guenther, M.; Gerlach, G.; Wallmersperger, T.; Avula, M.N.; Cho, S.H.; Xie, X.; Devener, B.V.; Solzbacher, F.; Tathireddy, P.; Magda, J.J.; et al. Smart Hydrogel-based biochemical microsensor array for medical diagnostics. Adv. Sci. Tech. 2013, 85, 47-52. [CrossRef] 
6. Guenther, M.; Wallmersperger, T.; Gerlach, G. Piezoresistive chemical sensors based on functionalized hydrogels. Chemosensors 2014, 2, 145-170. [CrossRef]

7. Gerlach, G.; Guenther, M.; Suchaneck, G.; Sorber, J.; Arndt, K.-F.; Richter, A. Application of sensitive hydrogels in chemical and pH sensors. Macromol. Symp. 2004, 210, 403-410. [CrossRef]

8. Erfkamp, J.; Guenther, M.; Gerlach, G. Hydrogel-based piezoresistive sensor for the detection of ethanol. J. Sens. Sens. Syst. 2018, 7, 219-226. [CrossRef]

9. Wang, J.; Ward, M.D.; Ebersole, R.C.; Foss, R.P. Piezoelectric pH Sensors: AT-cut quartz resonators with amphoteric polymer films. Anal. Chem. 1993, 65, 2553-2562. [CrossRef]

10. Tanaka, T.; Fillmore, D.J. Kinetics of swelling of gels. J. Chem. Phys. 1979, 70, 1214. [CrossRef]

11. Yang, S.-C.; Hou, J.-L.; Finn, A.; Kumar, A.; Ge, Y.; Fischer, W.-J. Synthesis of multifunctional plasmonic nanopillar array using soft thermal nanoimprint lithography for highly sensitive refractive index sensing. Nanoscale 2015, 7, 5760-5766. [CrossRef] [PubMed]

12. Lee, K.L.; Chang, C.-C.; You, M.-L.; Pan, M.-Y.; Wei, P.-K. Enhancing the surface sensitivity of metallic nanostructures using oblique-angle-induced fano resonances. Sci. Rep. 2016, 6, 33126. [CrossRef] [PubMed]

13. Piliarik, M.; Šípová, H.; Kvasnička, P.; Galler, N.; Krenn, J.R.; Homola, J. High-resolution biosensor based on localized surface plasmons. Opt. Express 2012, 20, 672-680. [CrossRef] [PubMed]

14. Zalyubovskiy, S.J.; Bogdanova, M.; Deinega, A.; Lozovik, Y.; Pris, A.D.; An, K.H.; Hall, W.P.; Potyrailo, R.A. Theoretical limit of localized surface plasmon resonance sensitivity to local refractive index change and its comparison to conventional surface plasmon resonance sensor. J. Opt. Soc. Am. 2012, 29, 994-1002. [CrossRef] [PubMed]

15. Arndt, K.-F.; Kuckling, D.; Richter, A. Application of sensitive hydrogels in flow control. Polym. Adv. Technol. 2000, 11, 496-505. [CrossRef]

16. Nishiyama, Y.; Satoh, M. Swelling behavior of poly(acrylic acid) gels in aqueous ethanol—effects of counterion species and ionic strength. Macromol. Rapid Commun. 2000, 21, 174-177. [CrossRef]

17. Bai, W.; Gariano, N.A.; Spivak, D.A. Macromolecular amplification of binding response in superaptamer hydrogels. J. Am. Chem. Soc. 2013, 135, 6977-6984. [CrossRef] [PubMed]

18. Mayer, K.M.; Hafner, J.H. Localized surface plasmon resonance sensors. Chem. Rev. 2011, 111, 3828-3857. [CrossRef] [PubMed]

19. Dmitriev, A. Nanoplasmonic Sensors; Springer: New York, NY, USA, 2012.

20. Svedendahl, M.; Chen, S.; Dmitriev, A.; Kall, M. Refractometric sensing using propagating versus localized surface plasmons: A direct comparison. Nano Lett. 2009, 9, 4428-4433. [CrossRef] [PubMed]

21. Çaykara, T.; Dogmus, M. The effect of solvent composition on swelling and shrinking properties of poly(acrylamideco-itaconic acid) hydrogels. Eur. Polym. J. 2004, 40, 2605-2609. [CrossRef]

22. Guenther, M.; Gerlach, G. Hydrogels for Chemical Sensors. In Hydrogel Sensors and Actuators-Engineering and Technology, Springer Series on Chemical Sensors and Biosensors 6; Springer: Berlin/Heidelberg, Germany, 2009; pp. 165-195.

(C) 2019 by the authors. Licensee MDPI, Basel, Switzerland. This article is an open access article distributed under the terms and conditions of the Creative Commons Attribution (CC BY) license (http://creativecommons.org/licenses/by/4.0/). 